\title{
STUDIES OF A DIRECT-CURRENT MICRODISCHARGE BASED MINIATURIZED PLASMA THRUSTER
}

\author{
U. KC, J. Bingaman, P. L. Varghese, and L. L. Raja
}

\begin{abstract}
Presented are the results of preliminary investigations of a direct-current microdischarge based miniaturized plasma thruster called a Microdischarge Plasma Thruster (MPT). The MPT has a triode configuration consisting of electrodes made of molybdenum separated by mica dielectric with argon propellant. Several variations of the triode were studied in order to determine a configuration that provided a relatively stable configuration. The thruster can produce an intense plume with a bluish outline. Current-voltage characteristics of the thruster are reported. Preliminary spectral measurements reveal that the plume emission arises from excited ground states of argon, with no emission from argon ions detected.
\end{abstract}

\section{INTRODUCTION}

Small satellites ( $<100 \mathrm{~kg}$ mass) have several potential benefits when compared to more traditional large satellites. In particular, so-called nanosatellites (with total mass $\sim 10 \mathrm{~kg}$ and linear dimensions $<50 \mathrm{~cm}$ ) are gaining much attention owing to their relatively low cost to build and operate, simplicity of design, rapid integration and testing, and the potential for formation flying and multivehicle operations [1]. Realizing these benefits requires the development and demonstration of enabling subsystem technologies and their integration into a nanosatellite system package. The principal challenge in the design and development of nanosatellite subsystems is the severe mass, volume, and power constraints posed by the overall size of the satellite. While significant recent advances in miniaturization of electronics, navigation, communication, sensor, and other relevant equipment have enabled new nanosatellite missions to be considered, the lack of appropriate propulsion devices for these satellites continues to be a major stumbling block.

The nanosatellite propulsion system is expected to have significantly lower thrust requirements than a conventional thruster, while conforming to the extremely constrained mass, volume, and power requirements. Thrusts range

This is an Open Access article distributed under the terms of the Creative Commons Attribution-Noncommercial License 3.0, which permits unrestricted use, distribution, and reproduction in any noncommercial medium, provided the original work is properly cited. 
from $\sim 1 \mu \mathrm{N}$ for small impulse bit precision thrusting applications to $\sim 10 \mathrm{mN}$ for satellite slew maneuvers. While the net thrust levels are low, desired performance parameters such as specific impulses and propulsive efficiencies are expected to be similar to or greater than those demanded from large-scale thrusters. Most importantly, it is certain that a 10-kilogram class nanosatellite cannot afford to accommodate multiple thrusters to suit different mission requirements such as drag makeup, orbit raising, precision pointing, and rapid maneuvering. There is significant ongoing research in the space propulsion community to develop micropropulsion devices that can satisfy small satellite $(<100 \mathrm{~kg})$ propulsion needs, but options for $\sim 10 \mathrm{~kg}$ class nanosatellites are still very limited.

A number of miniaturized versions of large-scale thrusters have been proposed for small satellites. For example, in the electrostatic class of high-specific-impulse propulsion devices, miniature Hall thrusters [2,3] and ion thrusters $[4,5]$ in the 100 -watt range have been developed, but the power and volume requirements for these devices are still in excess of what can be accommodated in a nanosatellite system where bus powers do not exceed $\sim 10-50 \mathrm{~W}$. Electromagnetic micro pulsed plasma thrusters (microPPT) have also been developed with lower power requirements $(\sim 10 \mathrm{~W})$ but the peak pulse voltage requirements of several kilovolt can be a design problem for a miniature power processing unit (PPU) in a nanosatellite [6]. Other more exotic thruster concepts have also emerged. For example, a field emission electric propulsion (FEEP) thruster has been developed with very high specific impulse (up to 10,000 s) and very low thrust levels $(\sim 10 \mu \mathrm{N})$ [7]. The FEEP thruster is suitable for the lower end nanosatellite thrusting requirements (e.g., long time scale drag makeup, precision pointing), but the very high voltage $(\sim 10 \mathrm{kV})$ can stretch the limits of a nanosatellite PPU and the liquid metal propellant (e.g., Cs) can pose a contamination hazard. Another example is a colloid thruster with performance characteristics similar to an FEEP thruster, i.e., high specific impulses $(\sim 1000 \mathrm{~s})$, low thrust $(\sim 100 \mu \mathrm{N})$, and high voltage requirements (several kilovolt) [8]. These thrusters can therefore be used for low thrust - high specific impulse mission requirements (e.g., drag makeup or small impulse bits for precision pointing), but have the same problems as FEEP devices in a nanosatellite application, i.e., high voltage requirements which stretch the PPU design.

For relatively high thrust requirements $(\sim 1-10 \mathrm{mN})$, such as for slew maneuvers and attitude control, scaled down versions of cold-gas thrusters [9], resistojets [10], combustion-based thrusters [11], and other variants are necessary. The problem of stabilizing combustion in micron-scale devices, storage of reactive propellants, and lifetime and preheating issues associated with catalysts when they are used, make combustion-based microthrusters less desirable for application in nanosatellites. Scaled down versions of resistojet and cold-gas thrusters with length scales $\sim 100 \mu \mathrm{m}$ are a viable choice for nanosatellite high thrust mission needs, but both suffer from low specific impulses ( $\sim 100 \mathrm{~s}$ or less) requiring the consumption of large propellant mass for a given mission $\Delta V$. 
In large satellite systems, a plasma-based arcjet thruster provides an efficient means of improving the specific impulse of an equivalent cold-gas thruster. However, downscaling an arcjet thruster to the micron-scale regime is hindered by two fundamental constraints: plasma breakdown and plasma quenching. The electrical breakdown of a gas can be understood in terms of the classical Paschen curve [12]; the breakdown voltage for most gases reaches a minimum value of $\sim 100 \mathrm{~V}$ for pressure-electrode distance product $(p d)$ values of $\sim 10$ Torr $\cdot \mathrm{cm}$. The breakdown voltage increases sharply for $p d$ values that are higher or lower. As the discharge dimensions decrease (lower $p d$ values), breakdown voltages become exceedingly high $(\sim 10 \mathrm{kV})$ and impractical to achieve. The second problem is one of quenching. The large surface-to-volume ratios in micron-scale geometries results in excessive loss of charged and active radical species to the bounding solid surfaces, making it difficult to sustain the plasma. Straightforward downscaling of plasma-based arcjet thruster devices is therefore not possible and alternative approaches must be explored.

This paper describes preliminary studies of a new type of thruster called an MPT that overcomes downscaling limitations of an arcjet and utilizes a directcurrent microdischarge plasma instead of an arc-like plasma. A direct-current (dc) microdischarge plasma is also used in Micro Hall Thrusters (MHT) [13], but it requires an external magnetic field and an external neutralizer, which are believed to be redundant for the MPT.

\section{MICRODISCHARGE PLASMA THRUSTER CONCEPT}

The MPT device provides a significant enhancement in performance relative to an equivalent cold-gas microthruster. The MPT geometry comprises two stages: the first being an upstream dc microdischarge stage and the second being a downstream ionization stage. As shown in Fig. 1, the dc microdischarge stage comprises electrodes $1\left(e_{1}\right)$ and $2\left(e_{2}\right)$ that are spaced apart using a dielectric $\left(m_{2}\right)$. Dielectric layer $1\left(m_{1}\right)$ is used to electrically isolate the MPT from the gas supply. The hole diameter for this stage is about $100 \mu \mathrm{m}$. This diameter is small enough to provide a sufficiently high pressure of about 100 Torr while maintaining a small flow rate of $\sim 1 \mathrm{sccm}(\sim 17 \mu \mathrm{L} / \mathrm{s})$ of the propellant gas. The downstream ionization stage comprises a larger hole diameter between $e_{2}$ and $e_{3}$. From a gas dynamic viewpoint, the MPT geometry resembles a converging-diverging micronozzle that accelerates a propellant gas stream to supersonic velocities. The gas dynamic acceleration in the diverging part of the geometry (ionization stage) results in a significantly lower pressure than in the first microdischarge stage. 


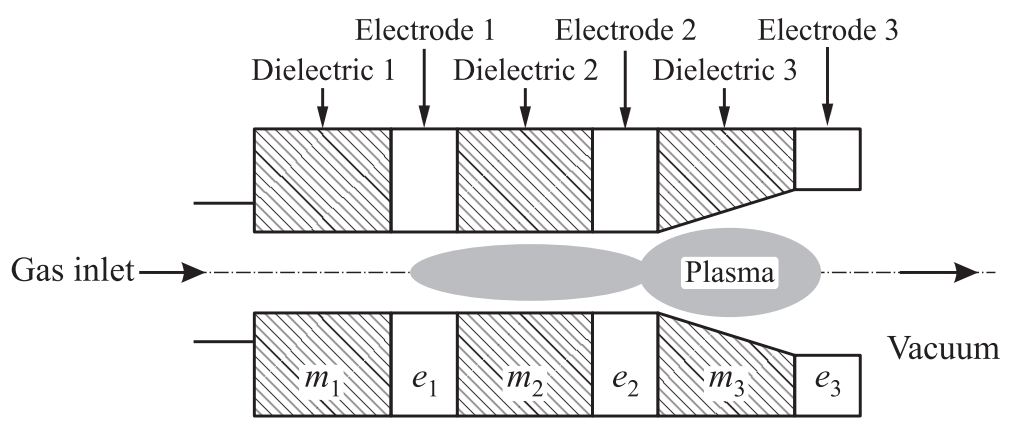

Figure 1 Schematic of the MPT concept

The overall operation of the MPT is as follows. The pressure between $e_{2}$ and $e_{3}$ is $\sim 100-1000$ mTorr. According to the Paschen curve [12], an extremely high voltage would be required to break down the gas inside a narrow channel at such low pressure. Nevertheless, if the microdischarge can be created in such a low pressure region then the ion content will be higher because the ratio $E / N$ of the characteristic electric field $(E)$ and neutral gas density $(N)$ is higher in the region. For this reason, the section between $e_{2}$ and $e_{3}$ is called the ionization stage. Higher ion fraction can enhance thrust since the ions are accelerated out of the MPT not only by gas dynamics but also by the electrical field. Thus, it is important to be able to break the gas between $e_{2}$ and $e_{3}$. The high-pressure region between $e_{1}$ and $e_{2}$ helps to lower the breakdown voltage, so that even in the low-pressure region, discharge can be stabilized at modest supply voltages. Just as in the microdischarge stage, the low-pressure ionization stage also relies on secondary electron emission processes at the third electrode $\left(e_{3}\right)$ to sustain the discharge phenomena. The discharge is created inside the MPT by setting $e_{1}$, $e_{2}$, and $e_{3}$ at different potentials.

Microdischarge phenomena in small diameter hollow electrode geometries at reasonably high pressures are now quite well understood. The microdischarge in a noble gas such as Ar results in charge densities of $\sim 10^{12}-10^{14} \mathrm{~cm}^{-3}$ although the ionization fraction at these charge densities is still very low $\left(\sim 10^{-6}-10^{-4}\right)$. The potential drop across the electrodes for the microdischarge stage is typically $\sim 300 \mathrm{~V}$ or higher. Overall, the ions produced by the microdischarge and the subsequent ionization stage are accelerated down the floating potential that exists between the MPT thruster and vacuum, resulting in a fraction of the total thrust being produced by the ions in addition to the gas dynamic (neutral) thrust produced by the discharge-heated neutral gas expanding through the converging-diverging nozzle geometry of the MPT.

In comparison to other microthruster concepts, e.g., cold-gas microthrusters, FEEP, MHT, etc., the MPT has advantages such as use of noncontaminat- 
ing/noncorrosive propellant gases, low dc voltage requirements, and absence of external magnetic fields or external electron emission sources.

\section{EXPERIMENTS}

\subsection{Test Facility}

The MPT was operated in a nonmagnetic stainless steel 6 way vacuum chamber approximately $0.15 \mathrm{~m}$ in diameter. The chamber was pumped using a Varian TV551 turbo molecular pump backed by an Alcatel 2015 SD general vane roughing pump. Pressure in the chamber was measured by HPS 943 digital Penning ionization cold-cathode gauge. It was mounted on the mouth of the chamber's upper extension that is perpendicular to the direction of gas flow and $0.25 \mathrm{~m}$ from the center of the chamber. Without Ar flow, this system achieves back pressures of the order of $10^{-6}$ Torr. When the Ar flow rate is between 1 and $5 \mathrm{sccm}$, the back pressure is of the order of $10^{-4}$ Torr. The flow rate is controlled by Omega FMA-2615A gas flow controller. The reservoir pressure is measured approximately $0.62 \mathrm{~m}$ upstream of the MPT by an MKS Baratron capacitance manometer. The line pressure is 0.9 Torr without Ar flow and it is between 10 and 100 Torr when the flow rate is between 1 and $5 \mathrm{sccm}(17-83 \mu \mathrm{l} / \mathrm{s})$.

\subsection{Microdischarge Plasma Thruster}

The MPT is illustrated schematically in Fig. 1. A photograph of the actual MPT is shown in Fig. 2. It has three layers of dielectric mica $\left(m_{1}, m_{2}\right.$, and $m_{3}$, respectively) and three layers of molybdenum $\left(e_{1}, e_{2}\right.$, and $e_{3}$, respectively) glued together with epoxy. The mica layers are each $25.4 \mathrm{~mm} \times 25.4 \mathrm{~mm} \times 200 \mu \mathrm{m}$ thick. The molybdenum electrodes $e_{1}$ and $e_{2}$ are $25.4 \times 6 \mathrm{~mm}$, and $e_{3}$ is 25.4 $\times 20 \mathrm{~mm}$. All three electrodes are 100-micron thick. A 100-micron diameter hole is drilled from $m_{1}$ to $m_{3}$. Configurations with 100 and $330 \mu \mathrm{m}$ holes in $e_{3}$ have been investigated. The MPT is mounted on a Lexan block. A 6-millimeter tube carrying Ar fits into the block. This was expected to lead to sonic flow at $m_{3}$ with a 330-micron diameter hole in $e_{3}$.

One of the objectives of the study was to examine how the degree of ionization between $e_{2}$ and $e_{3}$ depends on the diameter of the ionization region. Even in the configuration where the diameter of the bore between $e_{2}$ and $e_{3}$ is the same as in the upstream microdischarge region $(100 \mu \mathrm{m})$, the pressure is lower because the MPT is discharging to vacuum downstream, and hence, the degree of ionization in this region is expected to be higher than in the microdischarge region between $e_{1}$ and $e_{2}$. As the area ratio between the throat at $m_{3}$ and exit at $e_{3}$ is $1: 10.9$, the pressure in the microduct at $e_{3}$ is estimated to be of the order 


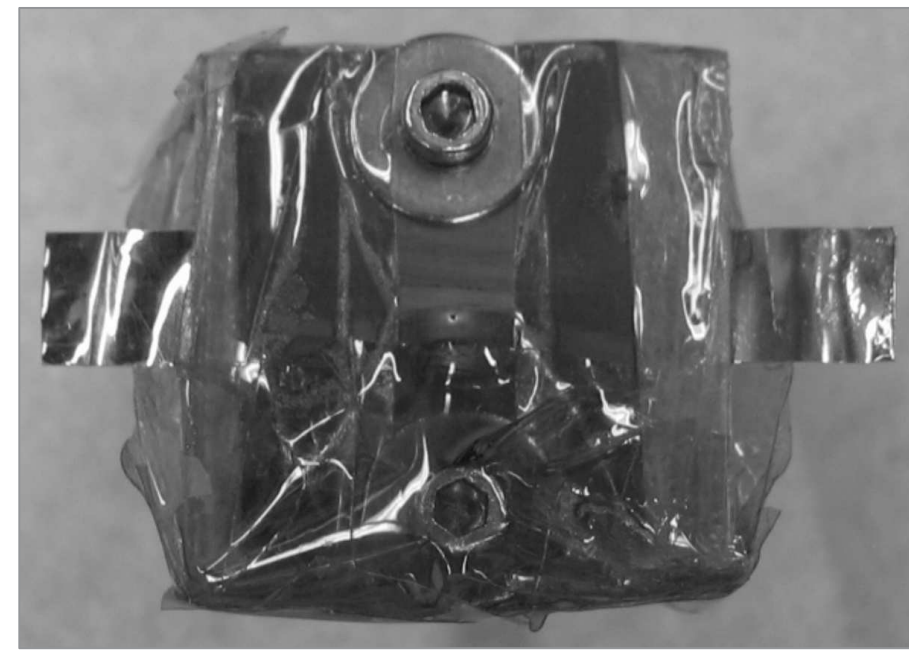

Figure 2 Front view of a partially insulated MPT attached to a Lexan block

of 1 Torr. It is not possible to strike a discharge in a 330-micron hole at gas pressures as low as 1 Torr with supply voltages of the order of a few hundred volts. However, this can be achieved by creating a pilot discharge between $e_{1}$ and $e_{2}$.

\subsection{Characterization}

A Matsusada AU-2P150-LC power supply was used to operate the MPT circuit. Emission spectroscopy of the plume was performed using a Jobin Yvon iHR 320 spectrometer fitted with a linear array detector at the output. Emission from the plume was collected orthogonally to the plume axis $\sim 4 \mathrm{~mm}$ downstream from the exit plane using a 75-millimeter diameter lens outside the vacuum chamber and focused into Thorlabs M28L01 optical fiber. The light transmitted through the fiber was focused into the spectrograph.

\section{RESULTS}

\subsection{Current-Voltage Characteristics}

The smallest breakdown voltage of $\mathrm{Ar}$ is obtained for the configuration that has $e_{1}, e_{2}$, and $e_{3}$ in decreasing order of potentials. The currents in the electrodes 


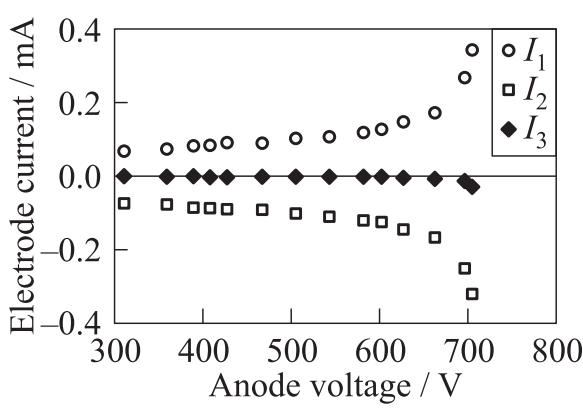

(a)

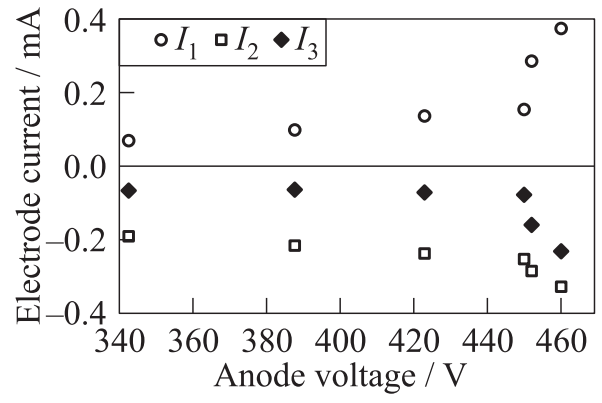

(b)

Figure 3 Current-voltage characteristics: $I_{1}, I_{2}$, and $I_{3}$ are the currents in $e_{1}, e_{2}$ and $e_{3}$, respectively; current entering the MPT is positive and that leaving it is negative; (a) represents the MPT with bores of 100 and $330 \mu \mathrm{m}$, and $(b)$ is for the MPT with a constant bore of $100 \mu \mathrm{m}$

are also stable at relatively low electrode potentials, and so this configuration is referred to as the stable configuration. In other configurations, e.g., potential increasing monotonically from $e_{1}$ to $e_{3}$, or $e_{2}$ as the anode and $e_{1}$ and $e_{3}$ as cathodes, even when the electrode potentials are relatively low, the discharge is highly unstable and cannot be sustained for even few seconds, so that it is impossible to record specific values of current and potential for a given supply voltage. For this reason, the MPT was studied only when operated in the stable configuration. The current-voltage characteristics when the plume is not visible, are shown in Fig. 3. In the case of the MPT with 100- and 330-micron holes, the voltage difference between $e_{1}$ and $e_{2}$ is 260 to $300 \mathrm{~V}$, and for the MPT with 100-micron hole, it varies from 180 to $250 \mathrm{~V}$. In both cases, $e_{3}$ is near ground potential. The current-voltage plots are only representative of the trend in current flow. One can speculate that when the gradient of the plot is slowly rising, the discharge forms only between $e_{1}$ and $e_{2}$ and there is almost no current in $e_{3}$. At higher anode voltages, the gas between $e_{2}$ and $e_{3}$ also breaks down which leads to a steep rise in current.

High power input to the discharge can change the properties of the dielectric. Initially, the electrodes are electrically insulated from each other by the mica dielectric sheets. However, after prolonged or multiple operations of MPT at high voltages, a nonzero conductance develops between the electrodes, presumably because of alteration of the dielectric. If the conducting path forms between $e_{1}$ and $e_{2}$ then it is no longer possible to create the microdischarge, whereas if the conductance forms between $e_{2}$ and $e_{3}$ then the current in $e_{3}$ jumps to $\sim 1 \mathrm{~mA}$ at some point as the electrode potentials are gradually increased. At this condition, a bright plume with bluish outline is observed outside the MPT and the currents in the electrodes fluctuate rapidly. The maximum fluctuation 


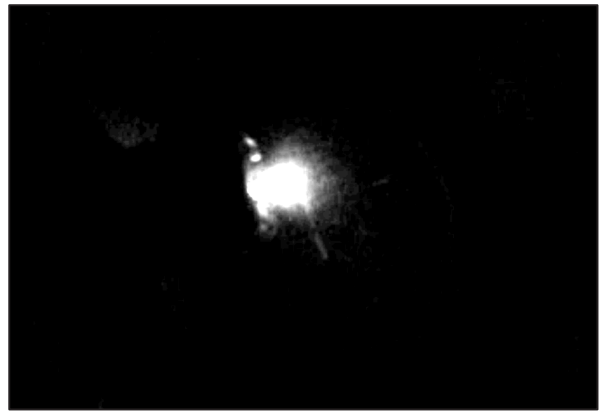

(a)

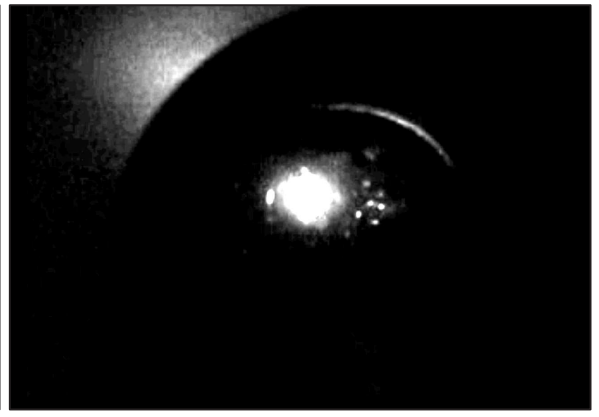

(b)

Figure 4 Side $(a)$ and front $(b)$ views of plume from an MPT that has diameter $100 \mu \mathrm{m}$ from $m_{1}$ to $m_{3}$ and $330 \mu \mathrm{m}$ from $m_{3}$ to $e_{3}$

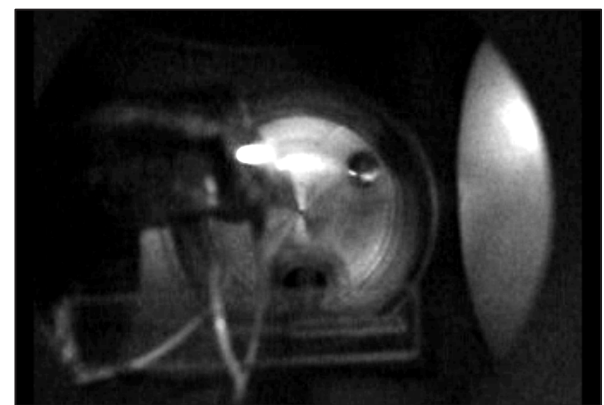

(a)

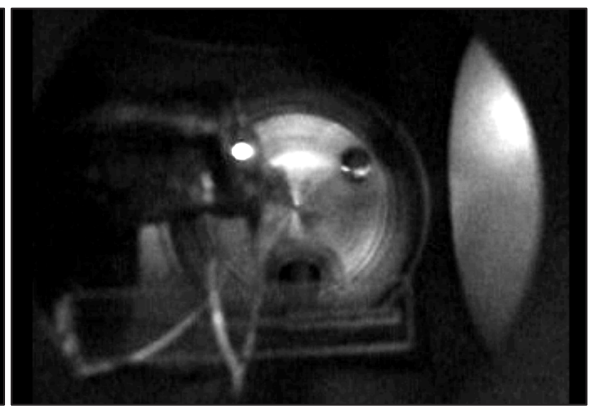

(b)

Figure 5 Side views of the MPT in the same configuration as Fig. 4. The two operating modes of the plume are seen: $(a)$ luminous extended plume; and $(b)$ compact plume with lower luminosity

is observed in $I_{3}$. The current has several alternate flowpaths: $(i)$ from $e_{1}$ to $e_{2}$ bypassing $e_{3},(i i)$ from $e_{1}$ directly to $e_{3}$ bypassing $e_{2}$, or $(i i i)$ from $e_{1}$ to both $e_{2}$ and $e_{3}$ in variable amounts. During operation when the plume is visible, the MPT operating mode seems to switch between the three modes which causes the plume to flicker, even in this nominally stable operating mode. Figures 4 to 6 illustrate the plumes observed. It is not possible to create an intense plasma by connecting external resistors to provide a conducting path between electrodes $e_{2}$ and $e_{3}$ of a new MPT. This shows that the formation of a conducting path alone does not explain the improvement in plume intensity. The MPTs producing plumes of Figs. 4 and 5 were observed in an MPT with internal conductance 
between $e_{2}$ and $e_{3}$, but the plume shown in Fig. 6 is observed in an MPT that has zero internal conductance. Furthermore, an intense plume is observed only after operating the MPT numerous times. Until then, only $I_{1}$ and $I_{2}$ increase with increasing source voltage, but $I_{3}$ remains low $(<0.1 \mathrm{~mA}$ approximately) as shown in Fig. 3.

The requirement that the MPT should run-in by operating a new device for several cycles indicates that these proof-of-concept experiments have not yet determined the opti-

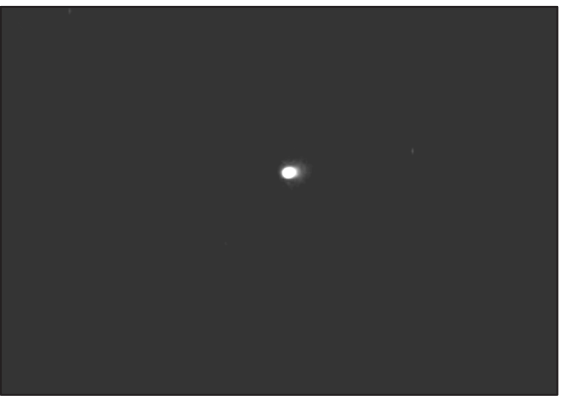

Figure 6 Side view of a mode of plume from an MPT with a constant bore of $100 \mu \mathrm{m}$ from $m_{1}$ to $e_{3}$ mum operating mode of an MPT.

A similar conclusion follows from the common but not invariable requirement that an internal conducting path between $e_{2}$ and $e_{3}$ should be established before one obtains an intense luminous external plume. Variables that need to be examined include dielectric and electrode material selection and MPT diameter variation. Additionally, better control of the experimental parameters is needed to prevent variables from changing during device operation. When the MPT was removed from the chamber and examined, it was found that one of the effects of the intense plume was the deposition of Mo dust on the insulation around the exposed area of $e_{3}$. Also, the size of the hole in $e_{3}$ was enlarged relative to its initial size. This shows that in these proof-of-concept experiments some of the parameters that should remain constant are not being controlled. The temperature of the MPT is another parameter that is not being controlled. These factors could make the plasma unstable.

\subsection{Spectral Measurements}

Preliminary spectra from the plume generated by the MPT with a constant bore of $100 \mu \mathrm{m}$ from $m_{1}$ to $e_{3}$ have been collected. The spectra were recorded with 10-second acquisition integration time and a spectrometer slit width of $100 \mu \mathrm{m}$.

Figure 7 shows parts of the spectrum. As expected, several prominent transitions of neutral Ar are observed, but no transitions from Ar ions were seen. While this is not conclusive, this leads one to believe that the gas emerging from the nozzle is only weakly ionized. The recorded spectra were compared to simulations of Ar spectra at Saha equilibrium from the NIST atomic spectra data base [14]. They are consistent with electron temperatures $\leq 0.7 \mathrm{eV}$ and electron densities approximately $10^{13} \mathrm{~cm}^{-3}$. 


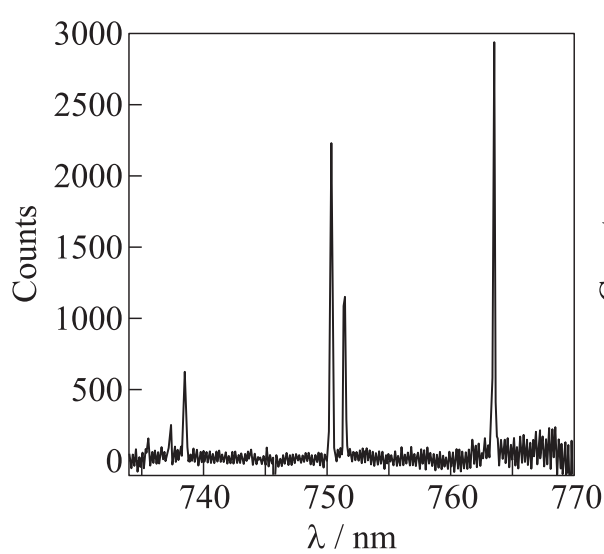

(a)

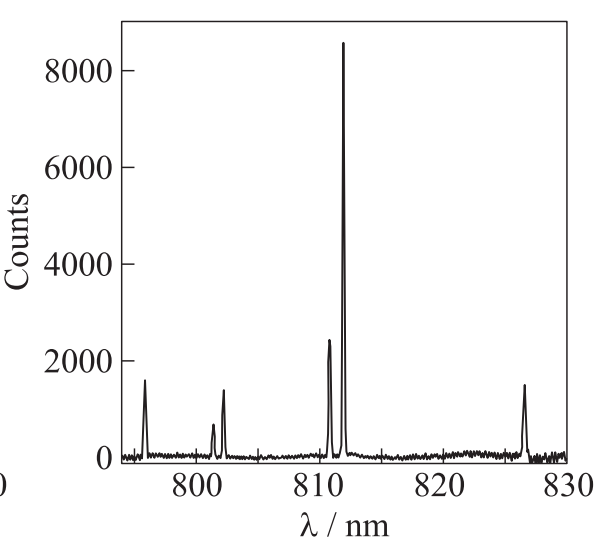

(b)

Figure 7 Spectra of the plume from an MPT with constant bore of $100 \mu \mathrm{m}$

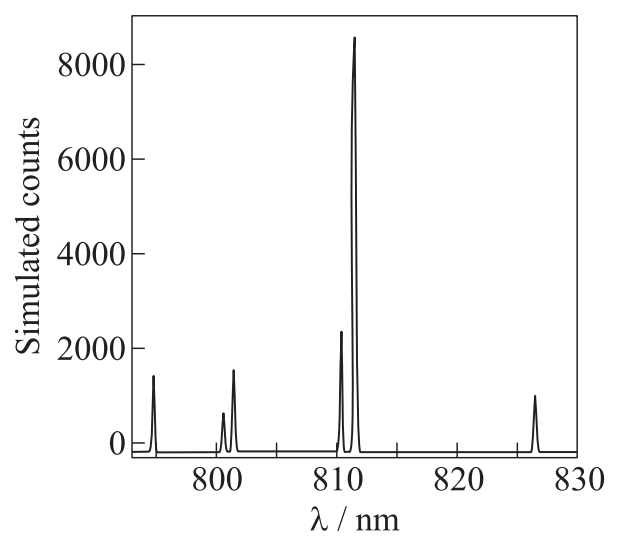

(a)

Figure 8 Simulated Ar spectrum between 794 and $830 \mathrm{~nm}$ with $T_{e}=0.7 \mathrm{eV}$ and $n_{e}$ $=10^{13} \mathrm{~cm}^{-3}$. The vertical axis has been scaled to match the counts of the strongest line in Fig. $7 b$ are strong $\mathrm{Ar}^{+}$transitions.
Figure 8 shows a simulated spectrum in the range $794-$ $830 \mathrm{~nm}$ computed for $T_{e}=0.7 \mathrm{eV}$ and $n_{e}=10^{13} \mathrm{~cm}^{-3}$. The simulation includes the Gaussian instrument function of actual spectrograph and detector (half-width of $0.11 \mathrm{~nm})$. The computed spectrum has been rescaled so that the maximum intensity matches the experimental value. It can be seen that the relative intensities of the spectral lines in the simulation are in reasonable agreement with the experimentally recorded spectrum. The ionization fraction is a sensitive function of electron temperature and number density assuming Saha equilibrium at these conditions. Additional measurements are planned near $440 \mathrm{~nm}$ where the spectrum is electron temperature because there 408 


\section{CONCLUDING REMARKS}

The characterization of the MPT is a work in progress. In this paper, preliminary results of current-voltage and spectral measurements were presented in proof-ofconcept experiments. In the nominally stable configuration, the plume is intense and bluish at high electrode potentials, but it cannot be sustained continuously. Very recently, it has been discovered that the most stable plume is produced when the potentials at $e_{1}$ and $e_{3}$ are set and $e_{2}$ is left floating. The plume produced in this configuration is spectrally similar to the ones produced for the cases discussed in this paper.

It is worth emphasizing that while the MPT appears to be a promising concept, its performance (thrust, specific impulse, and efficiency) must be measured experimentally in order to determine if it is a viable propulsion system for nanosatellites. The authors are currently building a torsion balance to measure the thrust produced by the MPT and thus assess its performance. Additionally, the group is currently working on a numerical model of the MPT to help determining the configuration that optimizes the device performance. In future work, the experimental setup will be modified and the MPT engineered so that experimental parameters, especially device temperature, are controlled during operation. Temperature control will enable tighter control of bore dimensions and electrical characteristics of the device during extended operation. In addition to the thrust measurements, spectroscopic measurements will be used to determine electron temperature and number density in the plume and a Langmuir probe will be used to measure the ion distribution in the plume.

\section{ACKNOWLEDGMENTS}

This work was supported by AFOSR under grant FA9550-06-1-0176.

\section{REFERENCES}

1. Lewin, A. 2004. Evaluating the present and potential future impact of small satellites. 18th Annual AIAA/USU Conference on Small Satellites.

2. Khayms, V., and M. Martinez-Sanchez. 1996. Design of a miniaturized Hall thruster for microsatellites. AIAA Paper No.96-3291.

3. Monheiser, J., V. Hruby, and C. Freeman. 2000. Development and testing of a low-power Hall thruster system. In: Micropropulsion for small spacecraft. Eds. M. M. Micci and A.D. Ketsdever. Progress in aeronautics and astronautics ser. 187: Ch. 10. 
4. Patterson, M. 1998. Low-power ion thruster development status. AIAA Paper No. 98-3347.

5. Gorshkov, O. 1998. Low-power Hall type and ion electric propulsion for small sized spacecraft. AIAA Paper No. 1998-3929.

6. Spangers, G. G., D. White, J. Schilling, S. Bushman, J. Lake, and M. Dulligan. 2001. AFRL microPPT development for the TechSat21 flight. 27th Electric Propulsion Conference (International). IEPC Paper No. 01-166.

7. Marcuccio, S., A. Genovese, M. Andrenucci, C. Bartoli, J. Gonzalez, and G. Saccocia. 1993. Field emission electric propulsion (FEEP) system study. 23rd Electric Propulsion Conference (International). IEPC Paper No. 93-156.

8. Martinez-Sanchez, M., and J. Pollard. 1998. Spacecraft electric propulsion - an overview. J. Propul. Power 14(5):688-99.

9. Bzibziak, R. 1992. Miniature cold gas thrusters.. AIAA Paper No. 92-3256.

10. Edwards, I., and R. E. W. Jansson. 1971. Gas dynamics of resistojets. J. Br. Interplanetary Soc. 24:729-42.

11. Barber, H.E., G.L. Falkenstein, C.A. Buell, and R. N. Gurnitz. 1971. Microthrusters employing catalytically reacted $\mathrm{N}_{2}-\mathrm{O}_{2}-\mathrm{H}_{2}$ gas mixtures, Tridyne. J. Spacecraft Rockets 8(2):111-16.

12. Raizer, Yu.P. 1991. Gas discharge physics. N.Y.: Springer, 1991.

13. Ito, T., N. Gascon, W. S. Crawford, and M. A. Cappelli. 2006. Further development of a micro Hall thruster. AIAA Paper No. 2006-4495.

14. Ralchenko, Yu., F.-C. Jou, D. E. Kelleher, A.E. Kramida, A. Musgrove, J. Reader, W. L. Wiese, and K. Olsen. 2007. NIST Atomic Spectra Database (version 3.12), [Online]. Available: http://physics.nist.gov/asd3 [2007, June 5]. National Institute of Standards and Technology, Gaithersburg, MD. 